\title{
Accurate Study of the Electromagnetic and Circuit Behavior of Finite Conducting Wedges and Interconnects with Arbitrary Cross-Sections
}

\author{
Thomas Demeester \\ Dept. of Information Technology \\ Ghent University, Gent, Belgium \\ Email: thomas.demeester@intec.ugent.be Email: dries.vandeginste@intec.ugent.be \\ Dries Vande Ginste \\ Dept. of Information Technology \\ Ghent University, Gent, Belgium
}

\author{
Daniël De Zutter \\ Dept. of Information Technology \\ Ghent University, Gent, Belgium \\ Email: daniel.dezutter@intec.ugent.be
}

\begin{abstract}
The manufacturing of interconnects often leads to conductors with a non-rectangular cross-section. Especially for sharp edges, it is therefore important to study the influence of corner effects on the interconnect circuit characteristics. Firstly, the electromagnetic behavior of a finite conducting 2-D wedge is investigated. Secondly, as an application example, a broadband transmission line model is used to study the influence of the conductors' shapes on the circuit behavior of a grounded coplanar waveguide. Both frequency and time domain results are presented.
\end{abstract}

\section{INTRODUCTION}

The challenges in the design and production of ever faster multi-gigabit interconnects are enormous. Electromagnetic wave effects and loss mechanisms are an important issue, not to mention many manufacturing difficulties of mechanical and chemical nature, related to the different materials. The problems of electromagnetic origin, such as wave phenomena, crosstalk, signal attenuation, dispersion, and heat production should be predicted and dealt with, by using accurate interconnect simulation software. As modern applications require high performance electronic circuitry, including high bandwidths, together with further miniaturization, an accurate knowledge of the behavior of metallic interconnects is of a paramount importance. Interconnects that are electrically long can often be formalized using a 2-D model of the actual configuration. Its behavior can then be expressed in terms of a traditional multi-conductor transmission line model (TLM), based on the resistance, capacitance, inductance, and conductance matrix per unit of length (p.u.l.).

Many transmission line models have been developed in the past (see the references in [1]). The model used in the simulations of this paper is described in [2]. This precise 2-D model takes both the (frequency-dependent) substrate loss mechanisms and the finite conductivity and shape of the conductors into account. The ever increasing complexity of the existing models, as the one used here, is dictated by the more stringent design specifications in terms of speed, loss mechanisms, bandwidth, etc. Accurate prediction of the manufacturing tolerances on the geometry also gains in importance.

We will focuss on the transmission line characteristics that are related to the lossy conductors' cross-section. From the

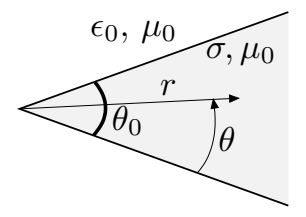

Fig. 1. Conducting wedge with opening angle $\theta_{0}$ and conductivity $\sigma$ placed in free space.

circuit perspective, a lot of work has been done in the past [3][8], especially for rectangular conductors. The field behavior near edges has also been under investigation for a long time, e.g., in [9] and [10]. The link with the circuit properties is however not obvious, as those papers only discuss the dominant singularity in the field expansion near corners. The tools for a more complete analysis have been developed in [7], [11], [12], which allow to accurately model finite conducting polygonal conductors up to deep skin effect frequencies, by means of the so-called Dirichlet-Neumann operator. Finally, [13] presents a more complete analysis, discussing the total field behavior near a wedge in combination with the singularity discussion of [10].

The aim of the current paper is twofold. Firstly, in Section II, we focus on the behavior near sharp edges, as in [13]. Here, however, we restrict ourselves to a comprehensive, yet graphical, overview of the behavior of the fields near the tip of a conducting wedge, as a function of its opening angle and frequency. Secondly, in Section III, it is shown that the developed numerical tools allow to investigate the effect of non-rectangular conductors on the interconnect level. In an application example, attention is devoted to finding out to what extent the circuit behavior of a grounded coplanar waveguide is sensitive to changes in the angles of the trapezoidal signal conductors. The line characteristics are investigated and illustrated in both the frequency and the time domain.

\section{FIELD BEHAVIOR NEAR EDGES}

Consider a nonmagnetic conducting wedge with conductivity $\sigma$ and opening angle $\theta_{0}$, as shown in Fig. 1. The purpose 


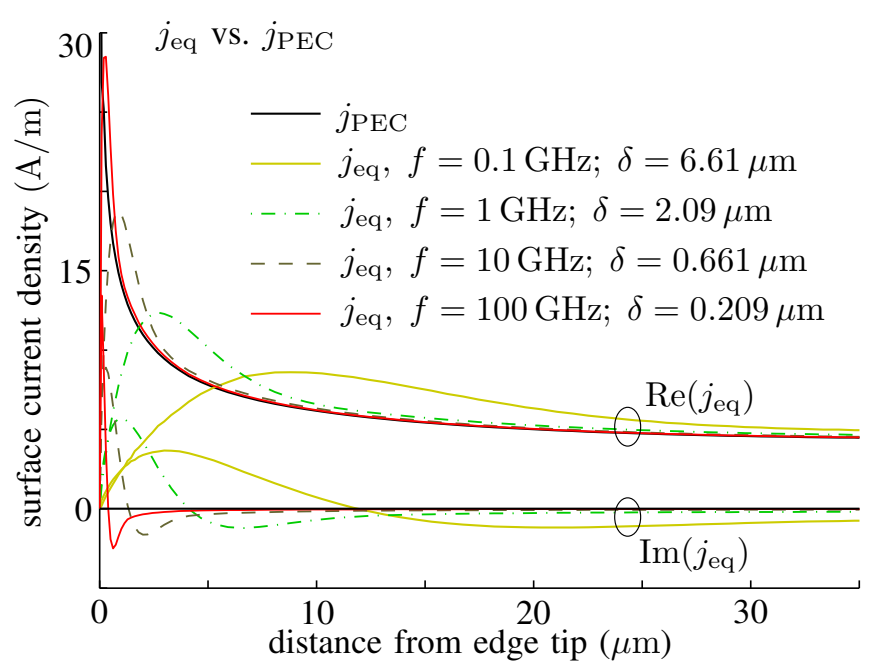

Fig. 2. $j_{\mathrm{PEC}}$ and the real and imaginary part of $j_{\text {eq }}$ along the edge of a copper wedge with opening angle $\theta_{0}=70^{\circ}$, for several frequencies and skin depths.

here is to provide some insight into the relevant phenomena for this single wedge, placed in a free-space background medium.

The effect of a conductor with a finite conductivity can be compared to its perfect electrically conducting (PEC) counterpart as follows. The perfect conductor carries a surface current density $\mathbf{j}_{\text {PEC }}$ such that the inner fields are zero. If the perfect conductor is removed and replaced by a surface current source equal to $\mathbf{j}_{\text {PEC }}$, the outer fields remain the same. For a finite conductor a similar procedure is possible. The conductor can be replaced by an equivalent surface current source $\mathbf{j}_{\text {eq }}$, residing in the background medium, that generates the same outside fields. For practical interconnects, the transverse dimensions are often much smaller than the wavelength of the fundamental propagation modes. In that case, the quasitransverse-magnetic (quasi-TM) approximations of Maxwell's equations hold [2], and $\mathbf{j}_{\mathrm{eq}}$ is directed in the longitudinal $z$ direction, viz. $\mathbf{j}_{\text {eq }}=j_{\text {eq }} \mathbf{u}_{z}$. The equivalent surface current density $j_{\text {eq }}$ is directly related to the cross-sectional tangential magnetic field component $h_{\tan }$ [7] and it is, by integration over the conductors' boundary, proportional to the inverse of the complex inductance matrix of the transmission line system. Hence, it is instructive to examine what exactly happens to $j_{\text {eq }}$ near a corner tip, compared to the PEC case. In Fig. 2 the real and imaginary part of $j_{\text {eq }}$ are shown at several frequencies, together with $j_{\mathrm{PEC}}$, along one of the sides of a copper wedge $(\sigma=58 \mathrm{MS} / \mathrm{m})$ with an opening angle of $\theta_{0}=70^{\circ}$. The simulations were performed for a triangular conductor in free space, put at a voltage of $1 \mathrm{~V}$. The sides of this triangle are very much larger than the skin depth such that over the major part of these sides (far enough away from the corners), this corner effect no longer plays a role.

The behavior of the perfect conductor surface current is singular, with $j_{\mathrm{PEC}} \propto r^{\pi /\left(2 \pi-\theta_{0}\right)-1}$ (with $r$ the distance from the corner tip, see Fig. 1), whereas $j_{\text {eq }}$ becomes zero for $r=0$. Nonetheless, $j_{\text {PEC }}$ appears to be the limiting case of $j_{\text {eq }}$ for
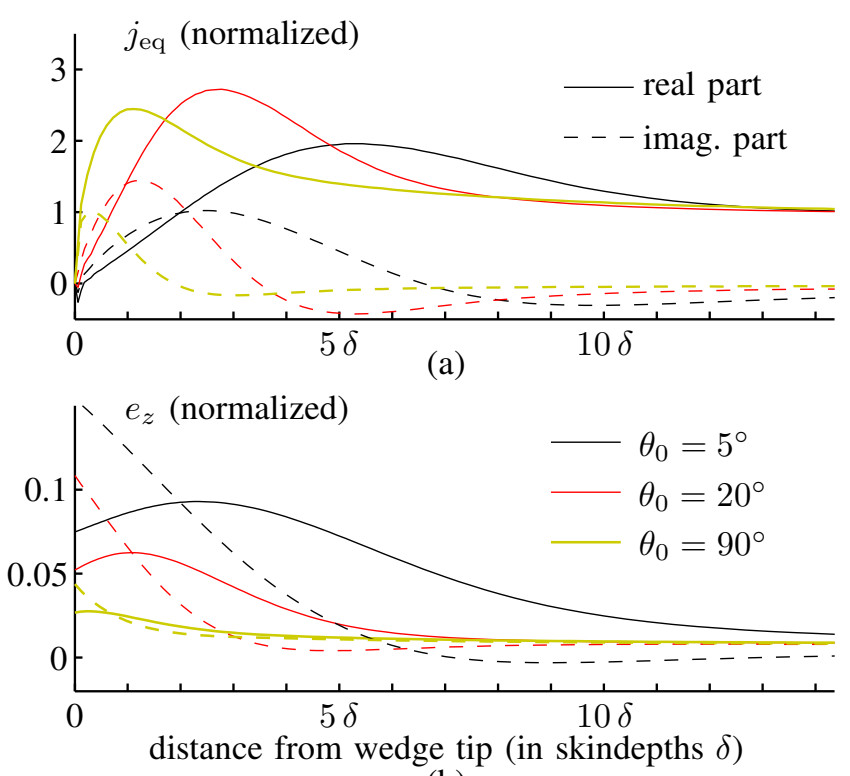

(b)

Fig. 3. (a) Real and imaginary part of $j_{\text {eq }}$ (normalized) along the edge of a copper wedge, for different values of $\theta_{0}$, and (b) real and imaginary part of $e_{z}$ (corresponding to the normalized current density). The abscissa is the distance to the wedge tip, expressed in skin depths $\delta$ at $f=1 \mathrm{GHz}(\delta=2.09 \mu \mathrm{m})$.

frequencies $f \rightarrow \infty$. Far enough away from the corner, $j_{\text {eq }}$ tends to a constant. Within a distance of several skin depths from the edge tip, however, the behavior is totally different. In particular, it can be observed that an important phase shift occurs, which has direct consequences on the losses induced by the conductor. Apart from the frequency, the extent of this transition region also depends on the opening angle. This is demonstrated in Fig. 3. The real and imaginary part of $j_{e q}$ are shown in Fig. 3 (a) for $f=1 \mathrm{GHz}$ (i.e. for a skin depth of about $2 \mu \mathrm{m}$ ), for different values of the opening angle $\theta_{0}$, and normalized to the constant value of $\left|j_{\text {eq }}\right|$, at a large distance from the corner. Clearly, the sharper the wedge, the larger the transition area around the tip. The longitudinal electric field $e_{z}$, corresponding with the normalized surface current shown in Fig. 3 (a), is displayed in Fig. 3 (b). For sharper corners, a strong increase in the electric field - and hence in the actual volume current density $j_{z}=\sigma e_{z}$ inside the conductor - is observed. For a $90^{\circ}$ angle, the influence of the corner is only present over a distance of about five skin depths.

\section{Application: Grounded Coplanar WaVEguide}

Consider the grounded coplanar waveguide (GCPW) shown in Fig. 4. The figure is on scale, and hence, only the neighborhood of the signal conductor can be shown. The copper planes at the left and the right of the signal conductor are reference conductors. The conductor at the bottom of the substrate is standing free. The conductors have a trapezoidal crosssection, which can be due to etching or other manufacturing processes. We assume symmetrical conductors, and the lower angles at each side of the conductors are denoted $\alpha$. The structure from Fig. 4 is designed to have a high-frequency 


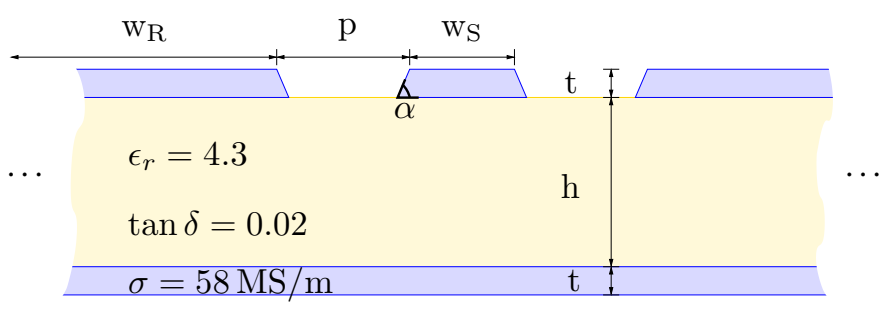

Fig. 4. Coplanar waveguide with $\mathrm{w}_{\mathrm{S}}=60 \mu \mathrm{m}, \mathrm{p}=80 \mu \mathrm{m}, \mathrm{w}_{\mathrm{R}}=800 \mu \mathrm{m}$, $\mathrm{t}=17 \mu \mathrm{m}$, and $\mathrm{h}=100 \mu \mathrm{m}$.

characteristic impedance of $75 \Omega$ when $\alpha=90^{\circ}$. In the following paragraphs, we will give some results, in which $\alpha$ varies from $45^{\circ}$ (smaller upper side), over $90^{\circ}$ (rectangular conductors), to $135^{\circ}$ (smaller lower side), but such that the top widths $\mathrm{w}_{\mathrm{S}}$ and $\mathrm{w}_{\mathrm{R}}$ of the conductors remain constant.

A varying angle $\alpha$ has a significant effect on the characteristic impedance $Z_{c}$ of the line, which, as a function of the p.u.l. parameters $R, L, G$, and $C$ of the line, is given by

$$
Z_{c}=\sqrt{\frac{j \omega L+R}{j \omega C+G}} .
$$

In Fig. 5, the real and imaginary part of $Z_{c}$ are shown for $\alpha=60^{\circ}, \alpha=90^{\circ}$, and $\alpha=120^{\circ}$. An angle of $\alpha=60^{\circ}$ leads to a smaller effective gap between the conductors. As a result, the capacitance $C$ is higher and the inductance $L$ is lower than in the rectangular conductor case. From (1), it can be expected that this leads to a lower $\operatorname{Re}\left(Z_{c}\right)$. This effect is illustrated in Fig. 5. The opposite effect is noticeable for $\alpha=120^{\circ}$. In Fig. $6, \operatorname{Re}\left(Z_{c}\right)$ is shown for $\alpha \in\left[45^{\circ}, 135^{\circ}\right]$ for several frequencies.

For a field-dependency $e^{-j \beta z}$ in the longitudinal $z$-direction, the propagation factor $\beta$ is determined by

$$
\beta^{2}=-(j \omega C+G)(j \omega L+R) .
$$

The effective relative permittivity $\epsilon_{r, \text { eff }}$ is found from $\beta$ by

$$
\epsilon_{r, \mathrm{eff}}=\left(\frac{\operatorname{Re}(\beta)}{k_{0}}\right)^{2}
$$

where $k_{0}=\omega / c$ is the free-space wavenumber (with $c$ the speed of light in vacuum). The phase velocity $v$ of the fundamental mode is given by $v=c / \sqrt{\epsilon_{r, \text { eff }}}$. Whereas the characteristic impedance (1) displays a large variability with $\alpha$, due to the opposite effect of $\alpha$ on $\mathrm{C}$ and $\mathrm{L}$ as explained above, from (2) and (3) it can be seen that $\alpha$ has less influence on $\epsilon_{r, \text { eff }}$, and thus on the phase velocity $v$. This is illustrated in Fig. 7, where $\epsilon_{r, \text { eff }}$ is shown as a function of $\alpha$. The attenuation factor of the fields, $e^{\operatorname{Im}(\beta) z}$, expressed in $\mathrm{dB} / \mathrm{mm}$, is also shown on Fig. 7. The same reasoning as for $\epsilon_{r, \text { eff holds, and }}$ so it is observed that the attenuation varies little with $\alpha$. E.g. at $10 \mathrm{GHz}$, for $\alpha=90^{\circ}$, the attenuation equals $0.039 \mathrm{~dB} / \mathrm{mm}$. For $\alpha=50^{\circ}$ the attenuation is $0.046 \mathrm{~dB} / \mathrm{mm}$ and for $\alpha=130^{\circ}$ the attenuation is $0.040 \mathrm{~dB} / \mathrm{mm}$.

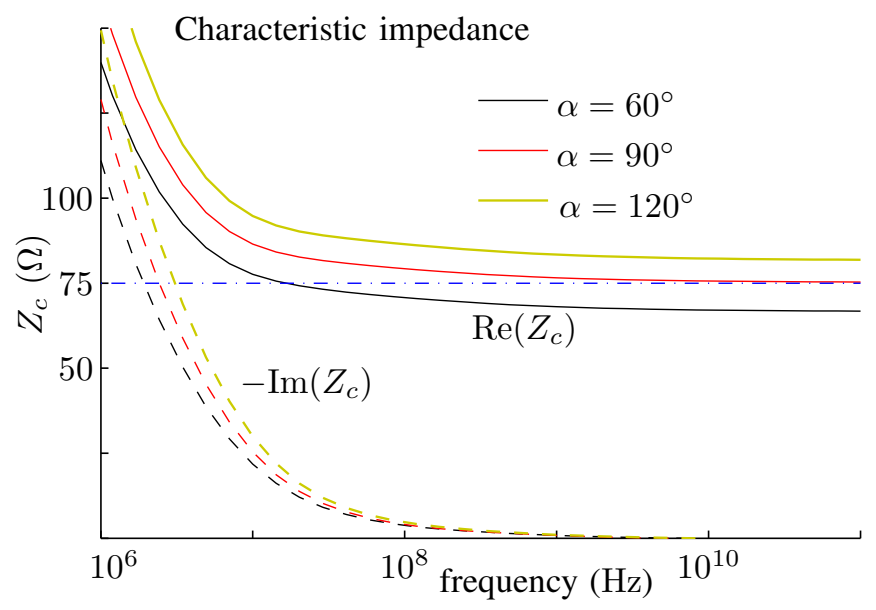

Fig. 5. Characteristic impedance $Z_{c}$ of the GCPW of Fig. 4 as a function of the frequency, shown for $\alpha=60^{\circ}, \alpha=90^{\circ}$ and $\alpha=120^{\circ}$. Full lines: $\operatorname{Re}\left(Z_{c}\right)$, dashed lines: $-\operatorname{Im}\left(Z_{c}\right)$.

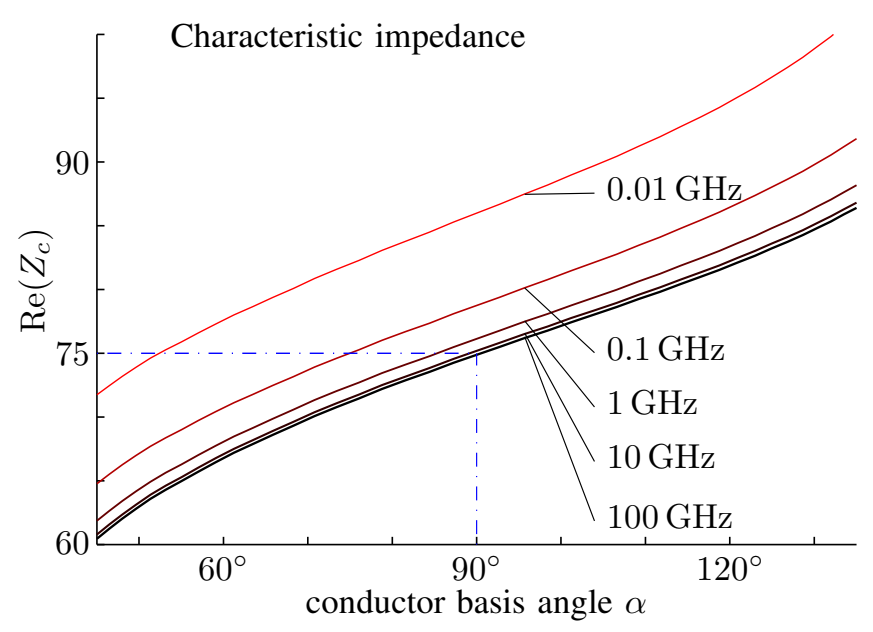

Fig. 6. Characteristic impedance $\operatorname{Re}\left(Z_{c}\right)$ of the GCPW of Fig. 4, shown as a function of the opening angle $\alpha$ for several frequencies. The dash-dot line represents the intended design of $75 \Omega$ for rectangular conductors.

To assess all effects induced by the varying cross-section simultaneously, we consider a transmission line with the GCPW topology of Fig. 4 and a length of $100 \mathrm{~mm}$. This line is driven by a time-domain voltage source with a voltage swing of $1 \mathrm{~V}$, bit period $100 \mathrm{ps}(10 \mathrm{~Gb} / \mathrm{s})$, and a rise and fall time of $30 \mathrm{ps}$. The driver and the load resistances are chosen $R_{S}=R_{L}=75 \Omega$. For three values of the angle $\alpha$, i.e. $50^{\circ}$, $90^{\circ}$, and $130^{\circ}$, the resulting time domain transmission (TDT) eye diagrams are shown in Fig. 8. It is observed that the eye opening is the largest in the case of $\alpha=90^{\circ}$, however, the results for $\alpha=50^{\circ}$ and $\alpha=130^{\circ}$ are quite similar, due to the effects described above. Although the characteristic impedance is very different in the three cases, the reflection at the load, i.e. $\frac{Z_{L}-Z_{c}}{Z_{L}+Z_{c}}$, remains below $10 \%$ in all three cases. Moreover, the vertical eye opening is largely dominated by the attenuation, which does not vary much as a function of $\alpha$ (see Fig. 7). Also, the phase velocity in all three cases is very similar. 


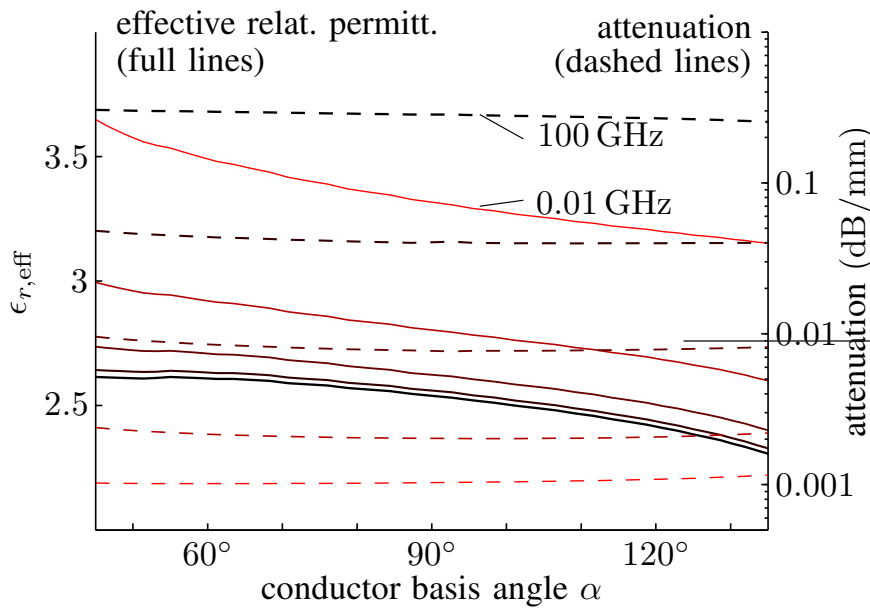

Fig. 7. Effective relative permittivity $\epsilon_{r, \text { eff }}$ and attenuation factor, as a function of $\alpha$, for the same frequencies as in Fig. 6. Increasing frequencies correspond with (i) decreasing $\epsilon_{r \text {,eff }}$ values (full lines) and (ii) increasing attenuation (dashed lines).

\section{Conclusion}

In this contribution, firstly, the behavior of the electromagnetic field along a 2-D finite conducting wedge was investigated for varying frequencies and trapezoidal conductor opening angles, showing a considerable difference with the perfect conductor case. Secondly, the circuit characteristics of a GCPW were investigated, again as a function of the frequency and the opening angle. In particular for this configuration, where the fields are concentrated near the central conductor and in the air gap between the conductor and the reference planes, the characteristic impedance of the line is quite sensitive to changes in the conductors' cross-section. However, TDT eye diagrams for a source-line-load configuration illustrate that the characteristic impedance does not compromise the time domain behavior of the line. So, a reliable interconnect can be achieved with the GCPW topology, despite possible manufacturing tolerances.

\section{REFERENCES}

[1] F. Olyslager, Electromagnetic Waveguides and Transmission Lines. Oxford, U.K.: Oxford University Press Inc., 1999.

[2] T. Demeester and D. De Zutter, "Quasi-TM transmission line parameters of coupled lossy lines based on the Dirichlet to Neumann boundary operator," IEEE Trans. Microw. Theory Tech., vol. 56, no. 7, pp. 16491660, Jul. 2008.

[3] M. Tsuk and J. Kong, "A hybrid method for the calculation of the resistance and inductance of transmission lines with arbitrary cross sections," IEEE Trans. Microw. Theory Tech., vol. 39, no. 8, pp. 13381347, Aug. 1991.

[4] K. Coperich, A. Ruehli, and A. Cangellaris, "Enhanced skin effect for partial-element equivalent-circuit (PEEC) models," IEEE Trans. Microw. Theory Tech., vol. 48, no. 9, pp. 1435-1442, Sep. 2000.

[5] W. Heinrich, "Comments on internal impedance of conductors of rectangular cross section," IEEE Trans. Microw. Theory Tech., vol. 49, no. 3, pp. 580-581, Mar. 2001.

[6] F. Medina, R. Marques, G. Antonini, A. Orlandi, and C. Paul, "Comments on internal impedance of conductors of rectangular cross section [and author's reply]," IEEE Trans. Microw. Theory Tech., vol. 49, no. 8, pp. 1511-1513, Aug. 2001

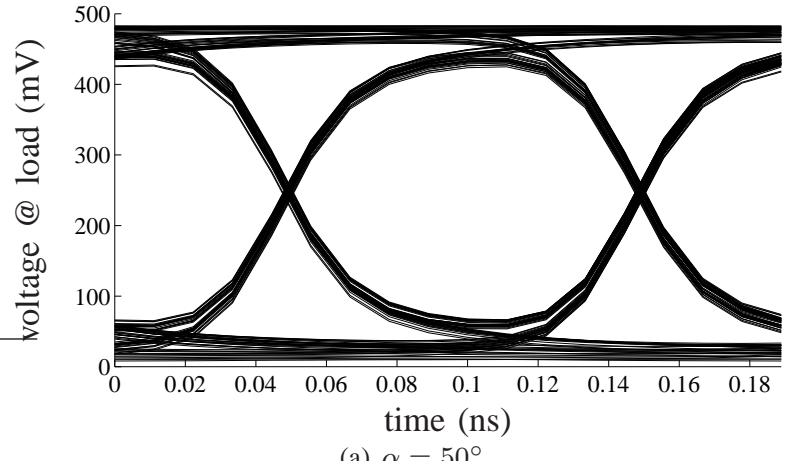

(a) $\alpha=50^{\circ}$

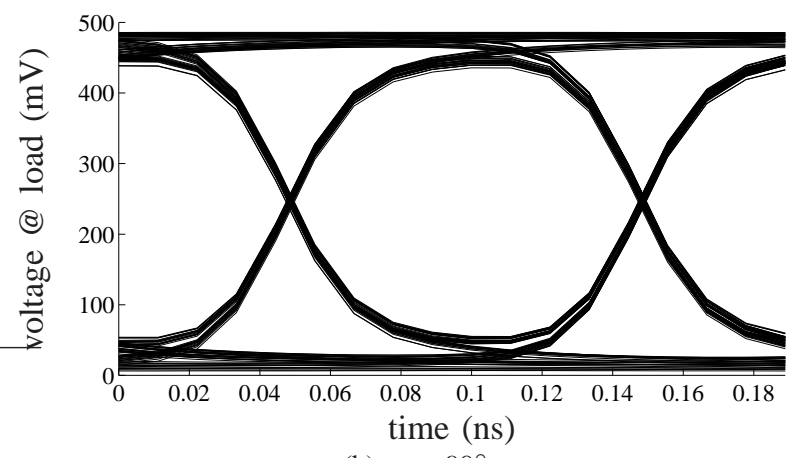

(b) $\alpha=90^{\circ}$

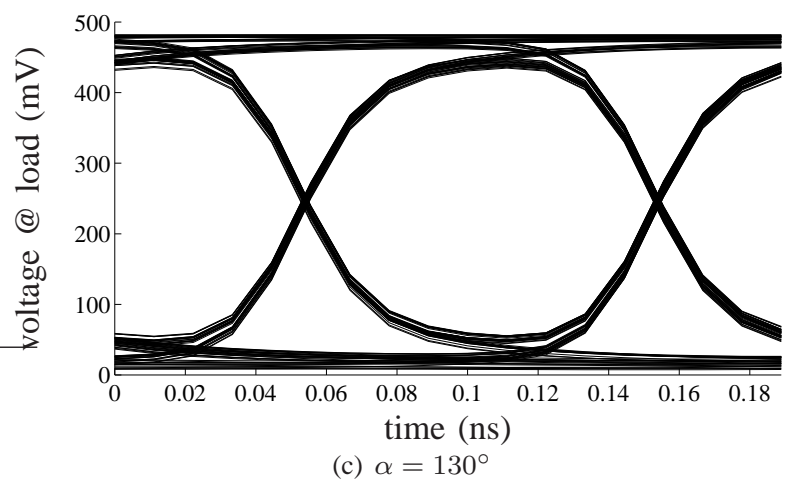

Fig. 8. TDT eye diagrams of a $100 \mathrm{~mm}$ line with the GCPW topology of Fig. 4 and for three values of $\alpha$.

[7] D. De Zutter and L. Knockaert, "Skin effect modeling based on a differential surface admittance operator," IEEE Trans. Microw. Theory Tech., vol. 53, no. 8, pp. 2526-2538, Aug. 2005.

[8] T. Demeester and D. De Zutter, "Modeling the broadband inductive and resistive behavior of composite conductors," IEEE Microw. Wireless Compon. Lett., vol. 18, no. 4, pp. 230-232, Apr. 2008.

[9] A. Maue, "Zur formulierung eines allgemeinen beugungs-problems durch eine integralgleichung," Zeitschrift für Physik A Hadrons and Nuclei, vol. 126, no. 7-9, pp. 601-618, Jul. 1949.

[10] J. Meixner, "The behavior of electromagnetic fields at edges," IEEE Trans. Antennas Propag., vol. 20, no. 4, pp. 442-446, Jul. 1972.

[11] T. Demeester and D. De Zutter, "Construction of the Dirichlet to Neumann boundary operator for triangles and applications in the analysis of polygonal conductors," IEEE Trans. Microw. Theory Tech., vol. 58, no. 1, pp. 116-127, Jan. 2010.

[12] — "Modeling the broadband resistive and inductive behavior of polygonal conductors," in International Conference on Electromagnetics in Advanced Applications, Torino, Italy, Torino, Italy, Sep. 2009.

[13] — - "Fields at a finite conducting wedge and applications in interconnect modeling," IEEE Trans. Microw. Theory Tech., accepted to be published in August, 2010. 